\title{
ON A CONJECTURE REGARDING NONSTANDARD UNISERIAL MODULES
}

\author{
PAUL C. EKLOF AND SAHARON SHELAH
}

\begin{abstract}
We consider the question of which valuation domains (of cardinality $\aleph_{1}$ ) have nonstandard uniserial modules. We show that a criterion conjectured by Osofsky is independent of $\mathrm{ZFC}+\mathrm{GCH}$.
\end{abstract}

\section{INTRODUCTION}

The story of the study of nonstandard uniserial modules is a long and interesting one, which we outline only briefly here; the reader may consult [BS1], [FS], or [O2] for more information. The existence of nonstandard uniserial modules over some valuation domains was first proved by the second author [Sh], by forcing a model of ZFC with a nonstandard uniserial module, and then using a completeness theorem for stationary logic to show that such examples exist in all models of ZFC, i.e., the existence is a consequence of ZFC. Then Fuchs and Salce [FS] constructed nonstandard uniserial modules using the diamond principle, which is consistent with ZFC. Fuchs noticed that a nonstandard divisible uniserial module could be used to construct an affirmative answer to Kaplansky's question whether there is a valuation ring which is not the quotient of a valuation domain. Fuchs and Shelah [FSh] used the compactness theorem for the logic with quantification over branches to give another proof that divisible nonstandard uniserials exist in all models of ZFC. The first author [E] elaborated on this argument to give a general transfer principle that, for example, showed that various different classes of nonstandard uniserials constructed by Bazzoni and Salce [BS1, 2, 3] using $\diamond$ existed in all models of ZFC.

Now this use of theorems from mathematical logic did not bother the second author; he was happy if algebraists had to learn logic! However, in this view he was probably a singleton among those interested in the problem. Aside from eliminating the methods of logic from the proof, the hope was that a more explicit proof would give more information, e.g. an understanding of exactly

Received by the editors September 10, 1991.

1991 Mathematics Subject Classification. Primary 13L05, 03E35, 13C05; Secondary 03E75, $13 \mathrm{~A} 18$.

Key words and phrases. Valuation domain, uniserial, nonstandard uniserial, Axiom(S), Gamma invariant.

The first author thanks Rutgers University for its research support through funding his visits to Rutgers.

The second author was partially supported by Basic Research Fund, Israeli Academy of Sciences Publ. No. 422. 
which valuation domains had nonstandard uniserial modules. Osofsky [O1,2] was the first to give a concrete construction (in $\mathrm{ZFC}$ ) of valuation domains with nonstandard uniserials. She ventured a conjecture [O1] as to which valuation domains had nonstandard uniserials; we will state it below in a modified form. It is a principal result of this paper that, while this conjecture is correct assuming $\mathrm{V}=\mathrm{L}$ (the Axiom of Constructibility), it fails in some other models of $\mathrm{ZFC}+\mathrm{GCH}$. Thus, it seems, logic cannot be expelled from the subject!

It is well known that if $R$ is "complete" in some sense (e.g., almost maxi$\mathrm{mal}$ ), then there are no nonstandard uniserial $R$-modules. Here we introduce a couple of invariants ("Gamma invariants") of a ring whose values are stationary subsets of $\omega_{1}$-or, more precisely, equivalence classes of stationary subsets under the equivalence relation of equality on a closed unbounded subset. These invariants can be viewed as measuring (the lack of) "completeness" of the ring. Some results about implications between the values of these invariants and the existence of nonstandard uniserials are proved in ZFC, but other implications are shown to be independent of ZFC.

We would like to thank Luigi Salce and Silvana Bazzoni for helpful comments.

\section{Preliminaries}

An $R$-module $U$ is called uniserial if its submodules are totally ordered by inclusion. An integral domain $R$ is a valuation domain if it is uniserial as an $R$-module. Throughout, $R$ will denote a valuation domain and $Q$ the quotient field of $R$; we assume $Q \neq R$. The uniserial modules we consider will always be generated by (at most) $\aleph_{1}$ elements, and most of the time $R$ will be of cardinality $\aleph_{1} . R^{*}$ denotes the set of units of $R$. The residue field of $R$ is defined to be $R / P$, where $P$ is the maximal ideal of $R$.

If $J$ and $A$ are $R$-submodules of $Q$ with $A \subseteq J$, then $J / A$ is a uniserial $R$-module, which is said to be standard. A uniserial $R$-module $U$ is said to be nonstandard if it is not isomorphic to a standard uniserial.

Given a uniserial module $U$, and a nonzero element, $a$, of $U$, let $\operatorname{Ann}(a)=$ $\{r \in R: r a=0\}$ and let $D(a)=\bigcup\left\{r^{-1} R: r\right.$ divides $a$ in $\left.U\right\}$. We say $U$ is of type $J / A$ if $J / A \cong D(a) / \operatorname{Ann}(a)$. This is well defined in that if $b$ is another nonzero element of $U$, then $D(a) / \operatorname{Ann}(a) \cong D(b) / \operatorname{Ann}(b)$. For example, $U$ has type $Q / R$ if and only if $U$ is divisible torsion and the annihilator ideal of every nonzero element of $U$ is principal. (But notice that there is no $a \in U$ with $\operatorname{Ann}(a)=R$.) It is not hard to see that if $U$ has type $J / A$, then $U$ is standard if and only if it is isomorphic to $J / A$.

Suppose $U$ has type $J / A$ and there exists $a \neq 0$ in $U$ such that $A=\operatorname{Ann}(a)$ and $J=D(A)$. Then

$$
J=\bigcup_{\nu<\omega_{1}} r_{\nu}^{-1} R
$$

for some sequence of elements $\left\{r_{\nu}: \nu<\omega_{1}\right\}$ such that for all $\mu<\nu, r_{\mu} \mid r_{\nu}$. If $J$ is countably generated, then $U$ is standard, so generally we will be assuming that $J$ is not countably generated; then it has a set of generators as in $(*)$, where, furthermore, $r_{\nu}$ does not divide $r_{\mu}$ if $\mu<\nu$, i.e., $r_{\nu}^{-1} r_{\mu}$ is not a member of $R$. Note that if $a_{\nu} \in U$ such that $r_{\nu} a_{\nu}=a$, then $\operatorname{Ann}\left(a_{\nu}\right)=r_{\nu} A$, 
so $a_{\nu} R(\subseteq U)$ is isomorphic to $R / r_{\nu} A=R /\left(A: r_{\nu}^{-1} R\right)$. If $\delta \in \lim \left(\omega_{1}\right)$ and

$$
J_{\delta} \stackrel{\text { def }}{=} \bigcup_{\nu<\delta} r_{\nu}^{-1} R
$$

then the submodule of $U$ generated by $\left\{a_{\nu}: \nu<\delta\right\}$ is a module over $R /\left(A: J_{\delta}\right)=R / \bigcap_{\nu<\delta} r_{\nu} A$.

Definition 1. A subset $C$ of $\omega_{1}$ is called a $c u b$-short for closed unbounded set-if $\sup C=\omega_{1}$ and for all $Y \subseteq C, \sup Y \in \omega_{1}$ implies $\sup Y \in C$. Call two subsets, $S_{1}$ and $S_{2}$, of $\omega_{1}$ equivalent iff there is a cub $C$ such that $S_{1} \cap C=S_{2} \cap C$. Let $\widetilde{S}$ denote the equivalence class of $S$. The inclusion relation induces a partial order on the set, $D\left(\omega_{1}\right)$, of equivalence classes, i.e., $\widetilde{S}_{1} \leq \widetilde{S}_{2}$ if and only if there is a cub $C$ such that $S_{1} \cap C \subseteq S_{2} \cap C$. In fact, this induces a Boolean algebra structure on $D\left(\omega_{1}\right)$, with least element, 0 , the equivalence class of sets disjoint from a cub; and greatest element, 1, the equivalence class of sets containing a cub. We say $S$ is stationary if $\widetilde{S} \neq 0$, i.e., for every cub $C, C \cap S \neq \varnothing$. We say $S$ is costationary if $\omega_{1} \backslash S$ is stationary.

Given $R$ and a type $J / A$, define $\Gamma_{R}(J / A)$ to be $\widetilde{S}$, where

$$
S=\left\{\delta \in \lim \left(\omega_{1}\right): R /\left(A: J_{\delta}\right) \text { is not complete }\right\}
$$

where the topology on $R /\left(A: J_{\delta}\right)$ is the metrizable linear topology with a basis of neighborhoods of 0 given by the submodules $r_{\nu} A(\nu<\delta) . \Gamma_{R}(J / A)$ is well defined in the sense that it does not depend on the choice of the sequence $\left\{r_{\nu}: \nu<\omega_{1}\right\}$, because of the following, which is proved by a standard argument (cf. [EM, pp. 85ff]).

Lemma 2. If $J=\bigcup_{\nu<\omega_{1}} r_{\nu}^{-1} R$ and also $J=\bigcup_{\nu<\omega_{1}} s_{\nu}^{-1} R$, then $\left\{\delta: \bigcap_{\sigma<\delta} r_{\sigma} A=\right.$ $\left.\bigcap_{\sigma<\delta} s_{\sigma} A\right\}$ is a cub.

Note that $\Gamma_{R}(J / A)=0$ if $J$ is countably generated.

If $J / A \cong J^{\prime} / A^{\prime}$, then $\Gamma_{R}(J / A)=\Gamma_{R}\left(J^{\prime} / A^{\prime}\right)$, but in general $\Gamma_{R}(J / A)$ and $\Gamma_{R}\left(J^{\prime} / A^{\prime}\right)$ will be different for different types $J / A$ and $J^{\prime} / A^{\prime}$. For example, if $R, A$ and $J$ are as in Example 2 of [BS1, p. 302], then $\Gamma_{R}(J / A)=1$, but $\Gamma_{R}(Q / A)=0$ (since $Q$ is countably generated). Note that if $R$ is almost maximal, then $\Gamma_{R}(J / A)=0$ for all types $J / A$.

In proving results about the existence or nonexistence of nonstandard uniserial modules it will be convenient to make use of the formulation of the problem given in [BS1], especially Lemma 1.2 and Test Lemma 1.3. Thus any uniserial module $U$ of type $J / A$ is described up to isomorphism by a family of units, $\left\{e_{\sigma}^{\tau}: \sigma<\tau<\omega_{1}\right\}$ such that

$$
e_{\tau}^{\delta} e_{\sigma}^{\tau}-e_{\sigma}^{\delta} \in r_{\sigma} A
$$

for all $\sigma<\tau<\delta<\omega_{1}$.

Moreover, if $U$ is given by $(\dagger)$, then $U$ is standard if and only if there exists a family $\left\{c_{\sigma}: \sigma<\omega_{1}\right\}$ of units of $R$ such that

$$
c_{\tau}-e_{\sigma}^{\tau} c_{\sigma} \in r_{\sigma} A
$$

for all $\sigma<\tau<\omega_{1}$. 
Salce has pointed out that by results of [BFS], the question of the existence of a nonstandard uniserial $R$-module of type $J / A$ can be reduced to the question of the existence of a nonstandard uniserial of type $K / R$ for an appropriate $K$.

\section{THE CONJECTURE}

We can paraphrase the conjecture of Osofsky in [O1, p. 164], for valuation domains $R$ of cardinality $\aleph_{1}$, as follows:

$R$ has a nonstandard uniserial of type $J / A$ if and only if $\Gamma_{R}(J / A)=1$.

Now, assuming $\mathrm{CH}$ (and the Axiom of Choice), it is possible to construct a valuation domain $R$ of cardinality $\aleph_{1}$ such that $\Gamma_{R}(J / A) \neq 0,1$ (see Definition 10 and the proof of Theorem 11). Thus the condition " $\Gamma_{R}(J / A)=1$ " cannot be necessary, because of the following result which implies that it is consistent with ZFC that there is a nonstandard uniserial $R$-module of type $J / A$ for such an $R$. Note that $\mho_{\omega_{1}}(S)$, for any stationary $S$, is consistent with ZFC; in fact it is implied by $\mathrm{V}=\mathrm{L}$ (cf. [D, p. 139]).

Proposition 3. Assume $R$ has cardinality $\aleph_{1}$.

(i) If $\Gamma_{R}(J / A)=\widetilde{S}$, then $\vartheta_{\omega_{1}}(S)$ implies that there exists a nonstandard uniserial $R$-module of type $J / A$.

(ii) If $\Gamma(J / A)=1$, then $C H$ implies that there exists a nonstandard uniserial $R$-module of type $J / A$.

Proposition 3(i) is essentially due to Fuchs and Salce [FS]; it can be proved, for example, by a straightforward generalization of the proof of Theorem 1.4 of [BS1], but for later purposes we will sketch the proof of a somewhat stronger result here. We begin with a lemma which, in one form or another, is a staple of the subject; its statement and proof, in our chosen notation, we include here for the sake of completeness.

Lemma 4. Suppose $\left\{e_{\sigma}^{\tau}: \sigma<\tau<\delta\right\}$ is a family of units satisfying ( $\dagger$ ) and $R / \bigcap_{\nu<\delta} r_{\nu} A$ is not complete. Then there are units $e_{\sigma, j}^{\delta}(\sigma<\delta ; j=0,1)$ such that

$$
e_{\tau, j}^{\delta} e_{\sigma}^{\tau}-e_{\sigma, j}^{\delta} \in r_{\sigma} A
$$

for all $\sigma<\tau$ and $j \in\{0,1\}$, but there is no sequence $\left\{c_{\sigma}: \sigma<\delta\right\}$ for which there are $c_{\delta, j} \in R^{*}(j=0,1)$ such that ( $\left.\dagger \dagger\right)$ holds for all $\sigma<\tau<\delta$ and moreover

$$
c_{\delta, j}-e_{\sigma, j}^{\delta} c_{\sigma} \in r_{\sigma} A
$$

for all $\sigma<\delta$ and $j \in\{0,1\}$.

Proof. Let $e_{\sigma, 0}^{\delta}$ be any family of units such that $e_{\sigma, 0}^{\delta}-e_{\tau, 0}^{\delta} e_{\sigma}^{\tau} \in r_{\sigma} A$ for all $\sigma<\delta$. Let $\left\langle v_{\sigma}: \sigma<\delta\right\rangle$ represent a Cauchy sequence in $R / \bigcap_{\nu<\delta} r_{\nu} A$ which does not have a limit in $R / \bigcap_{\nu<\delta} r_{\nu} A$. Let $e_{\sigma, 1}^{\delta}=v_{\sigma} e_{\sigma, 0}^{\delta}$. Then it is easy to check that $e_{\sigma, 1}^{\delta}-e_{\tau, 1}^{\delta} e_{\sigma}^{\tau} \in r_{\sigma} A$ for all $\sigma<\delta$. Moreover, if the conclusion of the lemma is contradicted by $\left\{c_{\sigma}: \sigma<\delta\right\}$ and $c_{\delta, 0}, c_{\delta, 1}$, then $c_{\delta, 1} c_{\delta, 0}^{-1}$ is a limit of $\left\langle v_{\sigma}: \sigma<\delta\right\rangle$, which is impossible.

Proof of Proposition 3 (sketch). In fact we will prove that, under the hypotheses on $R, \Phi_{\omega_{1}}(S)$ implies that there exists a nonstandard uniserial $R$-module of 
type $J / A$. (This generalizes the result in $[\mathrm{FrG}]$.) Here $\Phi_{\omega_{1}}(S)$ is the weak diamond principle which is implied by $\diamond_{\omega_{1}}(S)$ (see, for example, [EM, VI.1.6]). In [DSh] it is proved that $\mathrm{CH}$ implies $\Phi_{\omega_{1}}\left(\omega_{1}\right)$, so part (ii) follows.

Write $R$ as the union, $R=\bigcup_{\nu<\omega_{1}} R_{\nu}$, of a continuous chain of countable subsets. For each $\delta \in S$, define a function $P_{\delta}$ on pairs $(e, c)$ where $e=\left\{e_{\sigma}^{\tau}\right.$ : $\sigma<\tau<\delta\} \subseteq R_{\delta}, c=\left\{c_{\sigma}: \sigma<\delta\right\} \subseteq R_{\delta}$ as follows. If $e$ satisfies ( $\dagger$ ), let $\left\{e_{\sigma, j}^{\delta}: \sigma<\delta\right\}(j=0,1)$ be as in Lemma 4; if $e$ and $c$ satisfy ( $\dagger$ ) and there is $c_{0} \in R^{*}$ such that $c_{0}-e_{\sigma, 0}^{\delta} c_{\sigma} \in r_{\sigma} A$ for all $\sigma<\delta$, let $P_{\delta}(e, c)=1$; in all other cases let $P_{\delta}(e, c)=0$. Let $\rho: S \rightarrow 2$ be a weak diamond function (which predicts the values of the $P_{\delta}$ ). Now define the $e_{\sigma}^{\tau}$ by induction. The crucial case is when $\delta \in S$ and $e=\left\{e_{\sigma}^{\tau}: \sigma<\tau<\delta\right\}$ is contained in $R_{\delta}$; in this case let $e_{\sigma}^{\delta}=e_{\sigma, \rho(\delta)}^{\delta}$. After completing the inductive construction of the $e_{\sigma}^{\tau}$ for all $\sigma<\tau<\omega_{1}$, notice that for any sequence $\left\{c_{\sigma}: \sigma<\omega_{1}\right\}$, there is a cub $C$ such that for all $\delta \in C, e_{\sigma}^{\tau} \in R_{\delta}$ and $c_{\sigma} \in R_{\delta}$ for all $\sigma<\tau<\delta$. Then the construction and the properties of the weak diamond function imply that there is no sequence $\left\{c_{\sigma}: \sigma<\omega_{1}\right\}$ satisfying $(\dagger \dagger)$.

The condition $\Gamma_{R}(J / A) \neq 0$ is certainly necessary for the existence of a nonstandard uniserial $R$-module of type $J / A$, as the following easy lemma shows.

Lemma 5. If $\Gamma_{R}(J / A)=0$, then every uniserial $R$-module of type $J / A$ is standard.

Proof. Without loss of generality, we can assume that for all limit $\delta, R /\left(A: J_{\delta}\right)$ is complete. Given $\left\{e_{\sigma}^{\tau}: \sigma<\tau<\omega_{1}\right\}$ as in $(\dagger)$, we define the units $c_{\sigma}$ satisfying ( $\dagger \dagger)$ by induction on $\sigma$. If $c_{\sigma}$ has been defined, let $c_{\sigma+1}=e_{\sigma}^{\sigma+1} c_{\sigma}$. For a limit ordinal $\delta$, consider the Cauchy sequence in $R /\left(A: J_{\delta}\right)$ represented by the elements $\left\langle e_{\sigma}^{\delta} c_{\sigma}: \sigma<\delta\right\rangle$; this sequence has a limit which is represented by a unit of $R$, which we call $c_{\delta}$.

Thus we end up with the following reformulated version of the conjecture:

For any valuation domain $R$ of cardinality $\aleph_{1}, R$ has a nonstandard uniserial module of type $J / A$ if and only if $\Gamma_{R}(J / A) \neq$ 0 .

Theorem 6. Assuming $\mathrm{V}=\mathrm{L}$, this conjecture is true.

Proof. In fact, this follows from Proposition 3 and Lemma 5 since Gödel's Axiom of Constructibility, $\mathrm{V}=\mathrm{L}$, implies $\diamond_{\omega_{1}}(S)$ for all stationary $S$.

Thus the conjecture is consistent with $\mathrm{ZFC}$, and in fact with $\mathrm{ZFC}+\mathrm{GCH}$, since $\mathrm{V}=\mathrm{L}$ implies $\mathrm{GCH}$.

\section{ANOTHER GAMMA INVARIANT}

Let $R$ be a valuation domain of cardinality $\aleph_{1}$ and $J, A$ as before. For any limit ordinal $\delta<\omega_{1}$, let

$$
\mathscr{T}_{J / A}^{\delta}=\left\{\left\langle u_{\sigma}: \sigma<\delta\right\rangle: \forall \sigma<\tau<\delta\left(u_{\sigma} \in R^{*} \text {, and } u_{\tau}-u_{\sigma} \in r_{\sigma} A\right)\right\}
$$

that is, $\mathscr{T}_{J / A}^{\delta}$ consists of sequences of units which are Cauchy in the metrizable topology on $R /\left(A: J_{\delta}\right)$. Let $\mathscr{L}_{J / A}^{\delta}$ consist of those members of $\mathscr{T}_{J / A}^{\delta}$ which 
have limits in $R$, i.e.,

$$
\mathscr{L}_{J / A}^{\delta}=\left\{\left\langle u_{\sigma}: \sigma<\delta\right\rangle \in \mathscr{T}_{J / A}^{\delta}: \exists u_{\delta} \in R^{*} \text { s.t. } \forall \sigma<\delta\left(u_{\delta}-u_{\sigma} \in r_{\sigma} A\right)\right\} .
$$

Note that $\Gamma_{R}(J / A)=\tilde{S}$ where

$$
S=\left\{\delta \in \lim \left(\omega_{1}\right): \mathscr{T}_{J / A}^{\delta} \neq \mathscr{L}_{J / A}^{\delta}\right\}
$$

We will be making use of an $\omega_{1}$-filtration of $R$ by subrings, by which we mean an increasing chain $\left\{N_{\alpha}: \alpha \in \omega_{1}\right\}$ of countable subrings of $R$ such that $R=\bigcup_{\alpha \in \omega_{1}} N_{\alpha} ;$ and for limit $\alpha, N_{\alpha}=\bigcup_{\beta<\alpha} N_{\beta}$.

Definition. $\Gamma_{R}^{\prime}(J / A)=\widetilde{E}^{\prime}$ where

$$
\begin{gathered}
E^{\prime}=\left\{\delta \in \lim \left(\omega_{1}\right): \exists\left\langle u_{\sigma}: \sigma<\delta\right\rangle \in \mathscr{T}_{J / A}^{\delta} \text { s.t. } \forall\left\langle f_{\sigma}: \sigma<\delta\right\rangle \in \mathscr{L}_{J / A}^{\delta}\right. \\
\left.\exists \sigma<\delta \text { s.t. } u_{\sigma} f_{\sigma} \notin N_{\delta}\right\} \\
=\left\{\delta \in \lim \left(\omega_{1}\right): \exists\left\langle u_{\sigma}: \sigma<\delta\right\rangle \in \mathscr{T}_{J / A}^{\delta} \text { s.t. } \forall f \in R^{*} \exists \sigma<\delta\right. \\
\text { s.t. } \left.u_{\sigma} f \notin N_{\delta} \bmod r_{\sigma} A\right\} .
\end{gathered}
$$

As before, by standard methods it can be shown that the definition does not depend on the choice of $\left\{r_{\nu}: \nu<\omega_{1}\right\}$ or of $\left\{N_{\alpha}: \alpha<\omega_{1}\right\}$. (Here it is important that the $N_{\alpha}$ are countable.) Notice that $\Gamma_{R}^{\prime}(J / A) \leq \Gamma_{R}(J / A)$ since if $\mathscr{T}_{J / A}^{\delta}=\mathscr{L}_{J / A}^{\delta}$, then we can let $\left\langle f_{\sigma}: \sigma<\delta\right\rangle$ be $\left\langle u_{\sigma}^{-1}: \sigma<\delta\right\rangle$ to show $\delta \notin E^{\prime}$. We will prove below that the condition $\Gamma_{R}(J / A) \neq 0$ is not (provably from $\mathrm{ZFC}$ ) sufficient for the existence of a nonstandard uniserial of type $\mathrm{J} / A$; however, we do have the following theorem of ZFC.

Theorem 7. If $\Gamma_{R}^{\prime}(J / A) \neq 0$, then there is a nonstandard uniserial $R$-module.

Proof. Let $E^{\prime}$ be as above. It may be helpful to regard the following as a $\diamond\left(E^{\prime}\right)$-like argument carried out in ZFC, where the $N_{\delta}\left(\delta \in E^{\prime}\right)$ serve to give the predictions. We will construct the $e_{\sigma}^{\tau}(\sigma<\tau<\delta)$ satisfying $(\dagger)$ by induction on $\delta$ so that for every $\delta \in E^{\prime}$,

$$
\begin{aligned}
& \text { there is no sequence of units }\left\{c_{\sigma}: \sigma<\delta\right\} \subseteq N_{\delta} \text { such that there } \\
& \text { exists } c_{\delta} \in R^{*} \text { with } c_{\delta}-e_{\sigma}^{\delta} c_{\sigma} \in r_{\sigma} A \text { for all } \sigma<\delta .
\end{aligned}
$$

Suppose we can do this. We claim that the uniserial module given by the $e_{\sigma}^{\tau}$ is nonstandard. Indeed if not, there exists $\left\{c_{\sigma}: \sigma<\omega_{1}\right\}$ as in $(\dagger \dagger)$; then there is a cub $C$ such that for all $\delta \in C, c_{\sigma} \in N_{\delta}$ if $\sigma<\delta$; but then the construction is contradicted since $C \cap E^{\prime}$ is nonempty.

The definition of the $e_{\sigma}^{\tau}$ is routine except for the case when $\delta \in E^{\prime}$ and we are defining $e_{\sigma}^{\delta}$ assuming the $e_{\sigma}^{\tau}$ have been defined for $\sigma<\tau<\delta$. First choose any elements $\tilde{e}_{\sigma}^{\delta}$ so that $\left\{e_{\sigma}^{\tau}: \sigma<\tau<\delta\right\} \cup\left\{\tilde{e}_{\sigma}^{\delta}: \sigma<\delta\right\}$ satisfies (†). If (\#) is true, then we can let $e_{\sigma}^{\delta}=\tilde{e}_{\sigma}^{\delta}$. Otherwise, we have $\left\langle d_{\sigma}: \sigma<\delta\right\rangle \subseteq N_{\delta}$ such that there exists $d_{\delta} \in R^{*}$ with

$$
d_{\delta}-\tilde{e}_{\sigma}^{\delta} d_{\sigma} \in r_{\sigma} A \text { for all } \sigma<\delta .
$$

Let $\left\langle u_{\sigma}: \sigma<\delta\right\rangle$ be as in the definition of $E^{\prime}$ (i.e., there is no $f \in R^{*}$ such that for all $\sigma<\delta, u_{\sigma} f \in N_{\delta} \bmod r_{\sigma} A$ ). Let $e_{\sigma}^{\delta}=u_{\sigma} \tilde{e}_{\sigma}^{\delta}$. We claim (\#) is satisfied.

Suppose not; let $\left\{c_{\sigma}: \sigma<\delta\right\} \subseteq N_{\delta}$ such that there exists $c_{\delta} \in R^{*}$ with

$$
c_{\delta}-e_{\sigma}^{\delta} c_{\sigma} \in r_{\sigma} A \text { for all } \sigma<\delta \text {. }
$$


Let $f=c_{\delta}^{-1} d_{\delta}$. Fix $\sigma<\delta$ and let $\equiv$ denote congruence $\bmod r_{\sigma} A$. Then we have $c_{\delta} \equiv e_{\sigma}^{\delta} c_{\sigma} \equiv u_{\sigma} \tilde{e}_{\sigma}^{\delta} c_{\sigma}$ and $d_{\delta} \equiv d_{\sigma} \tilde{e}_{\sigma}^{\delta}$, so $f \equiv u_{\sigma}^{-1} c_{\sigma}^{-1} d_{\sigma}$. Thus $u_{\sigma} f \equiv$ $c_{\sigma}^{-1} d_{\sigma} \in N_{\delta}$, a contradiction of the choice of $\left\langle u_{\sigma}: \sigma<\delta\right\rangle$.

While the condition $\Gamma_{R}^{\prime}(J / A) \neq 0$ is thus sufficient for there to be a nonstandard uniserial $R$-module of type $J / A$, we shall see later (Corollary 15) that it is not necessary.

Let us say that a type $J / A$ is essentially uncountable if for every $\nu<\omega_{1}$ there exists $\mu>\nu$ such that $r_{\nu} A / r_{\mu} A$ is uncountable. This will be the case, for example, if $R$ has uncountable residue field, $J$ is not countably generated and $A=R$.

Theorem 8. $\left(\aleph_{1}<2^{\aleph_{0}}\right)$ If $R$ is a valuation domain of cardinality $\aleph_{1}$ and $J / A$ is essentially uncountable, then there is a nonstandard uniserial $R$-module of type $J / A$.

Proof. It suffices to prove that $\Gamma_{R}^{\prime}(J / A) \neq 0$; in fact we will show that $\Gamma_{R}^{\prime}(J / A)$ $=1$. Without loss of generality we can assume $J=\bigcup_{\nu<\omega_{1}} r_{\nu}^{-1} R$ where for all $\nu<\omega_{1}, r_{\nu} A / r_{\nu+1} A$ has cardinality $\aleph_{1}$. Let $R=\bigcup_{\alpha<\omega_{1}} N_{\alpha}$ be an $\omega_{1}$-filtration of $R$ by subrings. Let $\delta$ be a limit ordinal. Choose a ladder on $\delta$, i.e., a strictly increasing sequence $\gamma_{n}(n \in \omega)$ whose limit is $\delta$. We will define by induction on $n \in \omega$ a unit $u_{\eta}$ for each $\eta \in{ }^{n}$ - the set of all functions from $n=\{0,1, \ldots, n-1\}$ to $2=\{0,1\}$-such that $u_{\eta}-u_{\eta \dagger k} \in r_{\gamma_{k}} A$ for all $k<n$. If $u_{\eta}$ has been defined and $\eta_{i} \in{ }^{(n+1)} 2$ such that $\eta_{i} \mid n=\eta$ and $\eta_{i}(n)=i(i=0,1)$, then we choose $u_{\eta_{i}}$ congruent to $u_{\eta} \bmod r_{\gamma_{n}} A$ and such that $u_{\eta_{1}} u_{\eta_{0}}^{-1} \notin N_{\delta} \bmod r_{\gamma_{n+1}} A$. This is possible since $N_{\delta}$ is countable and $r_{\gamma_{n}} A / r_{\gamma_{n+1}} A$ is uncountable. (Indeed, choose $a \in A$ such that $r_{\gamma_{n}} a \not \equiv$ $y-1 \bmod r_{\gamma_{n+1}} A$ for any $y \in N_{\delta}$; then let $u_{\eta_{0}}=u_{\eta}$ and $u_{\eta_{1}}=\left(1+r_{\gamma_{n}} a\right) u_{\eta}$.) Thus for each of the $2^{\aleph_{0}}$ elements of $\zeta$ of $\omega_{2}$, we have a different family $\left\langle u_{\zeta \backslash n}: n \in \omega\right\rangle$. We claim that for at least one $\zeta$ there is no $f \in R^{*}$ such that for all $k, u_{\zeta \zeta k} f \in N_{\delta} \bmod r_{\gamma_{k}} A$-which will show $\delta \notin E^{\prime}$. Indeed, if there were no such $\zeta$, then since $R^{*}$ has cardinality $\aleph_{1}<2^{\aleph_{0}}$, there would be $\zeta_{0} \neq \zeta_{1}$ and $f$ satisfying

$$
u_{\zeta_{j} \nmid k} f \in N_{\delta} \quad \bmod r_{\gamma_{k}} A
$$

for all $k \in \omega$ and $j \in\{0,1\}$. Let $n$ be such that $\zeta_{0}\left|n=\zeta_{1}\right| n$ but $\zeta_{0}(n) \neq \zeta_{1}(n)$. It is then easy to obtain a contradiction of our construction of $u_{\eta_{i}}(i=0,1)$ if we let $\eta=\zeta_{0}\left\lceil n\right.$ and $\eta_{i}=\zeta_{i}\lceil(n+1)$.

Corollary 9. Let $R$ be a valuation domain of cardinality $\aleph_{1}$. If $J / A$ is essentially uncountable and $\Gamma_{R}(J / A)=1$, then there is a nonstandard uniserial $R$-module of type $J / A$.

Proof. If $\mathrm{CH}$ holds, this is by Proposition 3; if $\mathrm{CH}$ fails this is by Theorem 8.

We will deal with the essentially countable case in a later paper; in fact, Corollary 9 can be proved without the hypothesis of essential uncountability, but this requires a different construction in the essentially countable case. This 
means that Osofsky's original conjecture is vindicated for "natural" valuation domains, where $\Gamma_{R}(J / A)$ has to be either 0 or 1 .

\section{A CONSTRUCTION OF NONSTANDARD UNISERIALS}

As an application of Theorem 7, we will show how to construct nonstandard uniserials (of type $Q / R$ ) in ZFC over certain concretely given valuation domains $R$. (This may be regarded as a generalization of [O2] in that we define such $R$ with any given nonzero value for $\Gamma_{R}(Q / R)$.) We borrow an idea from [O2] (the definition of $u_{\sigma}$ ) in order to show that $\Gamma_{R}^{\prime}(Q / R)>0$. First we define the ring.

Definition 10. Let $G$ be the ordered abelian group which is the direct sum $\bigoplus_{\alpha<\omega_{1}} \mathbb{Z} \alpha$ ordered antilexicographically; that is, $\sum_{\alpha} n_{\alpha} \alpha>0$ if and only if $n_{\beta}>0$, where $\beta$ is maximal such that $n_{\beta} \neq 0$. (This is the group used in [FS] and [O2] and denoted $\Gamma\left(\omega_{1}\right)$ in [BS1, §3]). In particular, the basis elements have their natural order and if $\alpha<\beta$, then $k \alpha<\beta$ in $G$ for any $k \in \mathbb{Z}$.) Let $G^{+}=\{g \in G: g \geq 0\}$ and for each $\gamma<\omega_{1}$, let $G_{\gamma}^{+}$be the submonoid $\{g \in G: 0 \leq g<\gamma\}$ of $G^{+}$.

Fix a field $K$ of cardinality $\leq \aleph_{1}$ and let

$$
\widehat{R}=\left\{\sum_{y \in \Delta} k_{g} X^{g}: k_{g} \in K, \Delta \text { a well-ordered subset of } G^{+}\right\},
$$

$(=K[[G]]$; cf. [FS, pp. 24ff])

$$
\widehat{R}_{\alpha+1}=\left\{\sum_{g \in \Delta} k_{g} X^{g} \in \widehat{R}: \Delta \subseteq G_{\alpha}^{+}\right\}
$$

and for $\gamma$ a limit ordinal, $\widehat{R}_{\gamma}=\bigcup_{\alpha<\gamma} \widehat{R}_{\alpha}$.

Given an element $y=\sum_{g \in \Delta} k_{g} X^{g}$ of $\widehat{R}$, let $\operatorname{supp}(y)=\left\{g \in \Delta: k_{g} \neq 0\right\}$; let $p-\operatorname{supp}(y)=\left\{\alpha \in \omega_{1}: \exists g \in \operatorname{supp}(y)\right.$ whose projection on $\mathbb{Z} \alpha$ is nonzero $\}$. If $X \subseteq G$, then $y\left\lceil X\right.$ is defined to be $\sum_{g \in X \cap \Delta} k_{g} X^{g}$. Let $y|\alpha=y|\{g \in$ $G: g<\alpha\}$, the truncation of $y$ to $\alpha$. Note that $y \in \widehat{R}_{\alpha+1}$ if and only if $y=y\lceil\alpha$.

For $Y \subseteq \widehat{R}$, let $\langle Y\rangle_{\text {val }}$ denote the valuation subring generated by $Y$, i.e., the intersection with $\widehat{R}$ of the quotient field of the subring generated by $Y$.

For any stationary subset $S$ of $\omega_{1}$, let $R_{s}$ be the subring of $\widehat{R}$ consisting of all elements $y$ of $\widetilde{R}$ such that $\operatorname{supp}(y)$ is countable and no member of $S$ is a limit point of $p-\operatorname{supp}(y)$ (in the order topology).

Then $R_{S}$ is a valuation domain since $p-\operatorname{supp}\left(x y^{-1}\right) \subseteq p-\operatorname{supp}(x) \cup p-\operatorname{supp}(y)$. The cardinality of $R_{S}$ is $\leq 2^{\aleph_{0}}$.

Theorem 11. (CH) For every field $K$ of cardinality $\aleph_{1}$ and every stationary subset $S$ of $\omega_{1}$, there is a valuation domain $R$ of cardinality $\aleph_{1}$ with residue field $K$ and quotient field $Q$ such that $\Gamma_{R}(Q / R)=\widetilde{S}$ and there is a nonstandard uniserial $R$-module of type $Q / R$.

Proof. Let $R$ be $R_{S}$ as defined above. Let $r_{\sigma}=X^{\sigma}$, so that $Q=\bigcup_{\sigma<\omega_{1}} r_{\sigma}^{-1} R$. First notice that $\Gamma_{R}(Q / R) \geq \widetilde{S}$ since for any $\delta \in S \cap \lim \left(\omega_{1}\right)$, if $\gamma_{n}(n \in \omega)$ 
is a ladder on $\delta$ and $y_{n}$ is defined to be $\sum_{i=0}^{n-1} X^{\gamma_{i}}$ then $\left\{y_{n}: n<\omega\right\}$ is a Cauchy sequence in $R /\left(R: J_{\delta}\right)$ which does not have a limit, since a putative limit has $\delta$ as a limit point of its $p$-support. Moreover, $\Gamma_{R}(Q / R) \leq \widetilde{S}$ since for $\delta \notin S$, a Cauchy sequence in $R /\left(R: J_{\delta}\right)$ will have a limit in $R$ because $\delta$ is allowed to be the limit point of the $p$-support of an element of $R$.

To show that there is a nonstandard uniserial of type $Q / R$, it suffices by Theorem 7 to show that $\Gamma_{R}^{\prime}(Q / R) \geq \tilde{S}$. Let us fix an $\omega_{1}$-filtration of $R$ by subrings, $\left\{N_{\alpha}: \alpha \in \omega_{1}\right\}$. Let $\delta \in S$ and let $\left\langle\gamma_{n}: n \in \omega\right\rangle$ be a ladder on $\delta$. Let $L$ be the subfield of $K$ generated by the coefficients of the members of $N_{\delta}$. Then $L$ is a countable subfield of $K$ so we can inductively choose $t_{n} \in K \backslash L\left(t_{0}, \ldots, t_{n-1}\right)$. Let $u_{\sigma}=1+\sum_{i=0}^{n-1} t_{i} X^{\gamma_{i}}$ for all $\sigma$ with $\gamma_{n-1}<$ $\sigma \leq \gamma_{n}$. Then clearly $\left\langle u_{\sigma}: \sigma<\delta\right\rangle \in \mathscr{T}_{Q / R}^{\delta}$. It suffices to show that there is no $f \in R^{*}$ such that for all $\sigma<\delta, u_{\sigma} f \in N_{\delta} \bmod X^{\sigma} R$. Suppose there is such an $f$; by definition of $R_{S}$ and since $\delta \in S$, there exists $n$ such that $\sup (p-\operatorname{supp}(f \uparrow \delta)) \leq \gamma_{n-2}$. Say $f\left\lceil\delta=\sum_{g \leq \gamma_{n-2}} k_{g} X^{g}\right.$. But then, because of the properties of the value group-in particular because $2 \gamma_{n-2}<\gamma_{n-1}$-we see that the coefficient of $X^{\gamma_{n-1}}$ in $u_{\gamma_{n}} f$ is $t_{n-1} k_{0}$; similarly the coefficient of $X^{\gamma_{n}}$ in $u_{\gamma_{n+1}} f$ is $t_{n} k_{0}$. By assumption, these coefficients belong to $L$, and we easily obtain a contradiction of the choice of the $t_{i}$.

Remarks. (1) The hypothesis of $\mathrm{CH}$ was used only to insure that $R_{S}$ has cardinality $\aleph_{1}$. The ring defined by Osofsky in [O2] has cardinality $\aleph_{1}$ without invoking $\mathrm{CH}$, and the proof above applies to it. (It satisfies $\Gamma^{\prime}(Q / R)=1$.) Bazzoni has pointed out that the proof of Theorem 7 can be adapted to prove Theorem 11 for the rings $R_{S}$, even when $R_{S}$ has cardinality $2^{\aleph_{0}}>\aleph_{1}$ and so cannot have an $\omega_{1}$-filtration by countable subrings: choose an $\omega_{1}$-filtration of $K$ by countable subfields $L_{\alpha}$ and define $N_{\alpha}$ to be the subrings of elements whose coefficients come from $L_{\alpha}$.

(2) The main result of [O1] is very general in that it deals with arbitrary types, not just $Q / R$. Similarly the method of proof of Theorem 11 can be used to prove, in $\mathrm{ZFC}$, that there exist nonstandard uniserials of many other types than $Q / R$, e.g., ones like those given in Examples 1-6 of [BS1, pp. 301-305].

\section{$\operatorname{Axiom}(S)$}

As we have observed, Lemma 5 says that the condition $\Gamma_{R}(J / A) \neq 0$ is necessary for the existence of a nonstandard uniserial of type $J / A$. We now aim to prove that the condition $\Gamma_{R}(J / A) \neq 0$ is not provably sufficient if we assume only $\mathrm{GCH}$ (rather than $\mathrm{V}=\mathrm{L}$ ), i.e., the "if" direction of the conjecture fails in some model of $\mathrm{ZFC}+\mathrm{GCH}$. By Proposition 3 and Theorem 7, we will need to consider $R$ and $J / A$ where $\Gamma_{R}^{\prime}(J / A)=0$ and $\Gamma_{R}(J / A) \neq 1$. Analogies with the Whitehead Problem suggest the use of the following principle, known as $\operatorname{Ax}(S)$, which has been shown by the second author to be consistent with $\mathrm{ZFC}+\mathrm{GCH}$ (and with $\mathrm{ZFC}+\neg \mathrm{CH}$ ):

there is a stationary and costationary subset $S$ of $\omega_{1}$ such that for all proper posets $\mathbb{P}$ of cardinality $\aleph_{1}$ which are $\left(\omega_{1} \backslash S\right)$ complete, and for all families $\mathscr{D}$ of $\aleph_{1}$ dense subsets of $\mathbb{P}$, there is a $\mathscr{D}$-generic subset of $\mathbb{P}$.

(See [EM, pp. 170-173] for the necessary definitions.) 
We will be able to prove that in a model of $\mathrm{Ax}(S)+\mathrm{CH}$, there exists a valuation domain $R$ of cardinality $\aleph_{1}$ with $\Gamma_{R}(Q / R)=\widetilde{S}$ such that every uniserial of type $Q / R$ is standard.

Definition 12. For any countable subring $N$ of a valuation domain $R$ of cardinality $\aleph_{1}$, let

$$
\mathscr{T}_{J / A}^{\delta}(N)=\left\{\left\langle\bar{u}_{\sigma}: \sigma<\delta\right\rangle: \forall \sigma<\tau<\delta\left(u_{\sigma} \in N \text { and } u_{\tau}-u_{\sigma} \in r_{\sigma} A\right)\right\}
$$

where $\bar{u}_{\sigma}$ denotes the coset $u_{\sigma}+r_{\sigma} A$. Thus $\mathscr{T}_{J / A}^{\delta}(N)$ consists of sequences $\left\langle\bar{u}_{\sigma}: \sigma\langle\delta\rangle\right.$ where $\left\langle u_{\sigma}: \sigma\langle\delta\rangle \in \mathscr{T}_{J / A}^{\delta}\right.$ and all the $u_{\sigma}$ belong to $N$. Usually we shall abuse notation and write $\left\langle u_{\sigma}: \sigma<\delta\right\rangle$ for an element of $\mathscr{T}_{J / A}^{\delta}(N)$; but notice that $\bar{u}_{\tau}$ determines $\bar{u}_{\sigma}$ for all $\sigma<\tau$. Let

$$
\mathscr{L}_{J / A}^{\delta}(N)=\left\{\left\langle\bar{u}_{\sigma}: \sigma<\delta\right\rangle \in \mathscr{T}_{J / A}^{\delta}(N):\left\langle u_{\sigma}: \sigma<\delta\right\rangle \in \mathscr{L}_{J / A}^{\delta}\right\} .
$$

$\mathscr{T}_{J / A}^{\delta}(N)$ is given the tree topology, i.e., if $u=\left\langle\bar{u}_{\sigma}: \sigma\langle\delta\rangle \in \mathscr{T}_{J / A}^{\delta}(N)\right.$, a basis of neighborhoods of $u$ is the family $\left\{[u]_{\nu}: \nu<\delta\right\}$, where $[u]_{\nu}=\left\{\left\langle\bar{v}_{\sigma}: \sigma<\right.\right.$ $\delta\rangle \in \mathscr{T}_{J / A}^{\delta}(N): \bar{v}_{\sigma}=\bar{u}_{\sigma}$ for all $\left.\sigma \leq \nu\right\}$.

From now on $S$ always denotes the stationary and costationary subset of $\omega_{1}$ which is asserted to exist by $\operatorname{Ax}(S)$.

Theorem 13: $(\operatorname{Ax}(S))$ Suppose that $R$ is a valuation domain of cardinality $\aleph_{1}$ such that $\Gamma_{R}(J / A) \subseteq \widetilde{S}$ and $\Gamma_{R}^{\prime}(J / A)=0$. Suppose in addition that for some $\omega_{1}$-filtration of $R$ by subrings, $R=\bigcup_{\alpha \in \omega_{1}} N_{\alpha}$, the following holds for all $\delta \in S$ :

$$
\begin{aligned}
& \text { for every open subset } U \text { of } \mathscr{T}_{J / A}^{\delta}\left(N_{\delta}\right), U \cap \mathscr{L}_{J / A}^{\delta}\left(N_{\delta}\right) \text { is a non- } \\
& \text { meager (i.e., second category) subset of } \mathscr{T}_{J / A}^{\delta}\left(N_{\delta}\right) .
\end{aligned}
$$

Then every uniserial $R$-module of type $J / A$ is standard.

Proof. Let $U$ be a uniserial $R$-module of type $J / A$ given by a family $\left\{e_{\sigma}^{\tau}: \sigma<\right.$ $\left.\tau<\omega_{1}\right\}$ as in $(\dagger)$. Let the poset $\mathbb{P}$ consist of all sequences $p=\left\langle\bar{p}_{\sigma}: \sigma \leq \mu\right\rangle$ where $\mu$ is a countable ordinal (denoted $l(p)$ and called the length of $p$ ) such that

$$
\forall \sigma<\tau \leq \mu\left(p_{\sigma} \in R^{*} \text {, and } p_{\tau}-e_{\sigma}^{\tau} p_{\sigma} \in r_{\sigma} A\right) .
$$

The partial ordering is the natural one of extension of sequences. In an abuse of notation we shall write the elements of $\mathbb{P}$ as if they were sequences of elements of $R$ rather than sequences of cosets. Note that $\mathbb{P}$ is a tree and has the following properties:

(i) if $p, q \in \mathbb{P}, p_{\tau}=q_{\tau}$ for some $\tau$ and $c$ is a sequence of length $=l(q) \geq \tau$, such that $c_{\sigma} \equiv p_{\sigma}\left(\bmod r_{\sigma} A\right)$ for all $\sigma \leq \tau$, and $c_{\sigma} \equiv q_{\sigma}\left(\bmod r_{\sigma} A\right)$ for all $\tau \leq \sigma \leq l(q)$, then $c \in \mathbb{P}$;

(ii) if $p \in \mathbb{P}$ and $e \in R^{*}$, then $\left\langle e p_{\sigma}: \sigma \leq l(p)\right\rangle \in \mathbb{P}$.

For each $\nu<\omega_{1}$, let $D_{\nu}=\{p \in \mathbb{P}: p$ has length $\geq \nu\}$. Then $D_{\nu}$ is dense in $\mathbb{P}$ because given $q=\left\langle q_{\sigma}: \sigma \leq \mu\right\rangle \in \mathbb{P}$ (where we can assume $\mu<\nu$ ), define $p_{\sigma}=q_{\sigma}$ if $\sigma \leq \mu$ and $p_{\sigma}=\left(e_{\sigma}^{\nu}\right)^{-1} e_{\mu}^{\nu} q_{\mu}$ if $\mu \leq \sigma \leq \nu$. Then if $\sigma<\tau \leq \nu$, one may easily check that modulo $r_{\sigma} A$,

$$
p_{\tau}-e_{\sigma}^{\tau} p_{\sigma} \equiv\left(\left(e_{\tau}^{\nu}\right)^{-1}-e_{\sigma}^{\tau}\left(e_{\sigma}^{\nu}\right)^{-1}\right) e_{\mu}^{\nu} q_{\mu} \equiv 0 \cdot e_{\mu}^{\nu} q_{\mu}
$$


if $\mu \leq \sigma<\tau \leq \nu$, and similarly if $\sigma<\mu<\tau \leq \nu$. Thus $\left\langle p_{\sigma}: \sigma \leq \nu\right\rangle$ is an element of $\mathbb{P}$ extending $q$, so $D_{\nu}$ is dense in $\mathbb{P}$.

It suffices then to prove that there is a $\left\{D_{\nu}: \nu<\omega_{1}\right\}$-generic subset of $\mathbb{P}$; and for this, given the set-theoretic hypothesis, it suffices to prove that $\mathbb{P}$ is an $\left(\omega_{1} \backslash S\right)$-complete proper poset.

Let $\kappa$ be large enough for $\mathbb{P}$; let $\mathscr{C}$ be the cub of all countable elementary submodels $N$ of $\left(H(\kappa), \in, \omega_{1},\left\{e_{\sigma}^{\tau}: \sigma<\tau<\omega_{1}\right\}, R, \ldots\right)$ such that $N=$ $\bigcup_{i \in \omega} M_{i}$ where $M_{i} \prec M_{i+1}$ and $M_{i} \cap \omega_{1}<M_{i+1} \cap \omega_{1}$. Fix $N \in \mathscr{C}$ and let $\delta=N \cap \omega_{1}, \delta_{i}=M_{i} \cap \omega_{1}$. To show that $\mathbb{P}$ is $\left(\omega_{1} \backslash S\right)$-complete consider a chain $p^{0} \leq p^{1} \leq \cdots$ of elements $p^{n}=\left\langle p_{\sigma}^{n}: \sigma \leq l\left(p^{n}\right)\right\rangle$ of $\mathbb{P} \cap N$ such that for each dense subset $D$ of $\mathbb{P}$ which belongs to $\bar{N}$ there exists $n$ with $p^{n} \in D$. For all $n$ there exists $m_{n}$ such that $l\left(p^{m_{n}}\right) \geq \delta_{n}$ (because $\left.D_{\delta_{n}} \in N_{n+1}\right)$. For each $\sigma<\delta$, let $p_{\sigma}=p_{\sigma}^{m}$ for any $m$ such that $l\left(p^{m}\right) \geq \sigma$. Then $\left\{e_{\sigma}^{\delta} p_{\sigma}: \sigma<\delta\right\}$ is a Cauchy sequence in $R /\left(A: J_{\delta}\right)$, so if $\delta \notin S$, it has a limit $p_{\delta} \in R$, and then $\left\langle p_{\sigma}: \sigma \leq \delta\right\rangle$ is a member of $\mathbb{P}$ which extends each $p^{n}$.

So far, we have used just the hypothesis that $\Gamma_{R}(J / A) \leq \tilde{S}$. To show that $\mathbb{P}$ is proper, we need to use the additional hypotheses on $R$. Since the intersection of two cubs in a cub, we can assume, without loss of generality, that $N \cap R=$ $N_{\delta}$ (the $\delta$ th member of the $\omega_{1}$-filtration of $R$ given in the statement of the theorem).

Let $q \in \mathbb{P} \cap N$; we must find $p \geq q$ such that $p$ is $N$-generic. This is no problem if $\delta \notin S$ since $\mathbb{P}$ is $(\omega \backslash S)$-complete, so assume $\delta \in S$. Choose $d=\left\langle d_{\sigma}: \sigma \leq \delta\right\rangle \in \mathbb{P}$ of length $\delta$ and let $u_{\sigma}=e_{0}^{\delta}\left(e_{0}^{\sigma}\right)^{-1} d_{\sigma}$. Then $\left\langle u_{\sigma}: \sigma<\right.$ $\delta\rangle \in \mathscr{T}_{J / A}^{\delta}$. Since $\Gamma_{R}^{\prime}(J / A)=0$ we can assume that there exists $\left\langle f_{\sigma}: \sigma<\delta\right\rangle \in$ $\mathscr{L}_{J / A}^{\delta}$ with limit $f_{\delta}$ so that $u_{\sigma} f_{\sigma} \in N_{\delta}$ for all $\sigma<\delta$. Then, replacing $d$ by $\left\langle e_{0}^{\delta} d_{\sigma} f_{\sigma}: \sigma \leq \delta\right\rangle$, we can assume that $d_{\sigma}$ belongs to $N_{\delta}$ for all $\sigma<\delta$ (since $e_{0}^{\delta} d_{\sigma} f_{\sigma}=e_{0}^{\sigma} u_{\sigma} f_{\sigma}$; note that $e_{0}^{\sigma} \in N_{\delta}$ for all $\sigma<\delta$ ). Also, by (i) and (ii), we can assume that $d_{\sigma}=q_{\sigma}$ for all $\sigma \leq l(q)$. (Note that $l(q)<\delta$ since $q \in N$ and $N \cap \omega_{1}=\delta$.)

Let $U$ be the open subset

$$
\left\{\left\langle u_{\sigma}: \sigma<\delta\right\rangle \in \mathscr{T}_{J / A}^{\delta}\left(N_{\delta}\right): u_{\sigma}=1 \text { for } \sigma \leq l(q)\right\} .
$$

Given an element $u=\left\langle u_{\sigma}: \sigma<\delta\right\rangle$ of $\mathscr{T}_{J / A}^{\delta}\left(N_{\delta}\right)$, we will let $d u$ denote $\left\langle d_{\sigma} u_{\sigma}: \sigma<\delta\right\rangle$; notice that for all $\sigma<\tau<\delta$ we have

$$
d_{\tau} u_{\tau}-e_{\sigma}^{\tau} d_{\sigma} u_{\sigma}=d_{\tau} u_{\tau}-d_{\tau} u_{\sigma}+d_{\tau} u_{\sigma}-e_{\sigma}^{\tau} d_{\sigma} u_{\sigma} \in r_{\sigma} A
$$

so $d u$ belongs to $\mathbb{P}$. For each dense subset $D$ of $\mathbb{P}$ which belongs to $N$, let

$$
D^{\prime}=\left\{u \in \mathscr{T}_{J / A}^{\delta}\left(N_{\delta}\right): d u \text { is not an extension of a member of } D \cap N\right\} .
$$

Then $D^{\prime}$ is a nowhere dense subset of $\mathscr{T}_{J / A}^{\delta}\left(N_{\delta}\right)$ since for any basic open subset $[u]_{\nu}$, we can choose $u^{\prime}=\left\langle u_{\sigma}^{\prime}: \sigma \leq l\left(u^{\prime}\right)\right\rangle \geq\left\langle d_{\sigma} u_{\sigma}: \sigma \leq \nu\right\rangle$ which belongs to $D \cap N$, and then $\left\langle d_{\sigma}^{-1} u_{\sigma}^{\prime}: \sigma \leq l\left(u^{\prime}\right)\right\rangle$ determines a basic nonempty open subset of $[u]_{\nu}$ which is disjoint from $D^{\prime}$. (We are using here the fact that in the elementary submodule $N, D \cap N$ is a dense subset of $\mathbb{P} \cap N$; moreover note that $\left\langle\overline{d_{\sigma} u_{\sigma}}: \sigma \leq \nu\right\rangle$ belongs to $\mathbb{P} \cap N$.) Hence by $(\&)$, there is an element $w=\left\langle w_{\sigma}: \sigma<\delta\right\rangle$ of $U \cap \mathscr{L}_{J / A}^{\delta}$ which does not belong to $D^{\prime}$ for any $D \in \mathbb{P} \cap N$. Let $w_{\delta}$ be such that $w_{\delta}-w_{\sigma} \in r_{\sigma} A$ for all $\sigma<\delta$. Then $\left\langle d_{\sigma} w_{\sigma}: \sigma \leq \delta\right\rangle$ is the desired $N$-generic element extending $q$. 
Now the following result shows that the conjecture $(\$)$ fails in a model of $\mathrm{Ax}(S)+\mathrm{CH}$.

Theorem 14. $(\operatorname{Ax}(S)+\mathrm{CH})$ For any field $K$ of cardinality $\leq \aleph_{1}$, there is a valuation domain $R$ of cardinality $\aleph_{1}$ with residue field $K$ and a stationary, costationary subset $S$ of $\omega_{1}$ such that $\Gamma_{R}(Q / R)=\widetilde{S}$, but every uniserial $R$ module of type $Q / R$ is standard.

Proof. Let $\widehat{R}$ be as in Definition 10; it suffices to show there is a valuation subring $R$ of $\widehat{R}$ of cardinality $\aleph_{1}$ which satisfies the hypotheses of Theorem 13 for the type $Q / R$. We let $r_{\nu}=X^{\nu}$ which will be in $R$. (Thus $Q$ will be $\bigcup_{\nu<\omega_{1}} r_{\nu}^{-1} R$.) For each $\gamma \in \omega_{1}$, let $W_{\gamma}=\left\{y \in \widehat{R}_{\gamma}: \operatorname{supp}(y)\right.$ is finite and the coefficients of $y$ belong to the prime subfield of $K\}$.

We will now define by induction on $\alpha<\omega_{1}$ a continuous chain $\left\{Y_{\alpha}: \alpha<\right.$ $\left.\omega_{1}\right\}$ of valuation subrings of $\widehat{R}$ such that for all $\alpha: W_{\alpha} \subseteq Y_{\alpha} \subseteq \widehat{R}_{\alpha} ; Y_{\alpha} \cap R^{*}=$ $Y_{\alpha}^{*}$. Moreover, the $Y_{\alpha}$ will be closed under truncation, that is, if $y \in Y_{\alpha}$ and $\beta<\alpha$, then $y \mid \beta \in Y_{\beta+1}$. We will then define $R$ to be the union of the $Y_{\alpha}$.

First of all notice that each element of $\mathscr{T}_{Q / R}^{\delta}(N)$ is uniquely represented by a sequence $u=\left\langle u_{\sigma}: \sigma<\delta\right\rangle$ where for all $\sigma<\delta, u_{\sigma} \in N$, and $u_{\tau} \mid \sigma=u_{\sigma}$ whenever $\tau \geq \sigma$. We shall consider these to be the elements of $\mathscr{T}_{Q / R}^{\delta}(N)$. Moreover, given $u=\left\langle u_{\sigma}: \sigma\langle\delta\rangle \in \mathscr{T}_{Q / R}^{\delta}\left(Y_{\delta}\right)\right.$, there is a unique element of $\widehat{R}_{\delta+1}$, which we will denote $u_{\delta}$, which represents the limit of $\left\langle u_{\sigma}: \sigma\langle\delta\rangle\right.$ in $\widehat{R} / \bigcap_{\nu<\delta} r_{\nu} \widehat{R}$. If $u_{\delta} \notin Y_{\delta+1}$, then, by closure under truncation, the sequence $\left\langle u_{\sigma}: \sigma<\delta\right\rangle$ will not have a limit in $R$. Given a subset $Z$ of $\mathscr{T}_{Q / R}^{\delta}\left(Y_{\delta}\right)$, we will denote by $Z_{[\delta]}$ the set $\left\{u_{\delta}: u \in Z\right\} \subseteq \widehat{R}_{\delta+1}$.

Let $Y_{0}=\langle\{1\}\rangle_{\text {val }}$. Suppose that $Y_{\beta}$ has been defined for all $\beta<\gamma$. If $\gamma$ is a limit ordinal, let $Y_{\gamma}=\bigcup_{\beta<\gamma} Y_{\beta}$. If $\gamma=\delta+1$ and $\delta$ is a successor ordinal, let $Y_{\gamma}$ be the valuation subring of $\widehat{R}$ generated by $Y_{\delta} \cup W_{\delta+1}$. If $\delta \notin S$, let $Y_{\gamma}$ be the valuation subring generated by $Y_{\delta}$ together with the $u_{\delta}$ for each $u \in \mathscr{T}_{Q / R}^{\delta}\left(Y_{\delta}\right)$.

If $\delta$ is a limit ordinal in $S$, we must satisfy the hypotheses of Theorem 13 and make sure $R / \bigcap_{\nu<\delta} r_{\nu} R$ is not complete. Let $\left\{\left(N_{\beta}, \Phi_{\beta}, V_{\beta}\right): \beta<2^{\aleph_{0}}=\right.$ $\left.\aleph_{1}\right\}$ be an enumeration of all triples such that $N_{\beta}$ is a countable subring of $Y_{\delta}, V_{\beta}$ is a basic open set in $\mathscr{T}_{Q / R}^{\delta}\left(N_{\beta}\right)$, and $\Phi_{\beta}$ is the complement of a countable union of closed nowhere dense subsets of $\mathscr{T}_{Q / R}^{\delta}\left(N_{\beta}\right)$. (This enumeration is possible since each $\mathscr{T}_{Q / R}^{\delta}\left(N_{\beta}\right)$ has a countable base of open sets.) Also enumerate $\mathscr{T}_{Q / R}^{\delta}\left(Y_{\delta}\right)$ as $\left\{u^{\alpha}=\left\langle u_{\sigma}^{\alpha}: \sigma<\delta\right\rangle: \alpha<2^{\aleph_{0}}\right\}$. We will inductively define elements $y_{\alpha}$ of $\widehat{R}_{\delta+1}\left(\alpha<2^{\aleph_{0}}\right)$ and then let $Y_{\delta+1}=\left\langle Y_{\delta} \cup\left\{y_{\alpha}: \alpha<2^{\aleph_{0}}\right\}\right\rangle_{\text {val }}$. Fix an element $c=\left\langle c_{\sigma}: \sigma<\delta\right\rangle$ of $\mathscr{T}_{Q / R}^{\delta}\left(Y_{\delta}\right)$ and let $c_{\delta} \in \widehat{R}_{\delta+1}$ be its limit, as above. We also require that $c$ is chosen so that $c_{\delta} \notin \widehat{R}_{\delta}$. The elements $y_{\alpha}$ will be chosen with the following properties for all $\alpha<2^{\aleph_{0}}$ :

(i) if $\alpha=2 \beta+1$, then $y_{\alpha}=u_{\delta}$ for some $u \in \Phi_{\beta} \cap V_{\beta}$;

(ii) if $\alpha=2 \beta$, then $y_{\alpha}=f_{\delta}$ for some $f=\left\langle f_{\sigma}: \sigma<\delta\right\rangle \in \mathscr{T}_{Q / R}^{\delta}\left(Y_{\delta}\right)$ such that $u_{\sigma}^{\beta} f_{\sigma} \in W_{\delta}$ for all $\sigma<\delta$;

(iii) $c_{\delta} \notin\left\langle Y_{\delta} \cup\left\{y_{\gamma}: \gamma<\alpha\right\}\right\rangle_{\text {val }}$. 
Then (i) (resp. (ii)) will imply that (\&) holds (resp. $\Gamma_{R}^{\prime}(Q / R)=0$ ), and (iii) will insure that $R / \bigcap_{\nu<\delta} r_{\nu} R$ is not complete.

Suppose that $y_{\gamma}$ has been defined for all $\gamma<\alpha$ and that $\alpha=2 \beta+1$. Temporarily let $R^{\prime}=\left\langle Y_{\delta} \cup\left\{y_{\gamma}: \gamma<\alpha\right\}\right\rangle_{\text {val }}$. We claim that

there is a subset $Z$ of $\Phi_{\beta} \cap V_{\beta}$ of cardinality $>1$ such that $Z_{[\delta]}$ is algebraically independent over $R^{\prime}$.

If so, there exists $u \in Z$ such that $c_{\delta} \notin\left\langle R^{\prime} \cup\left\{u_{\delta}\right\}\right\rangle_{\text {val }}$ and then we can let $y_{\alpha}=u_{\delta}$. For otherwise, we have $u \neq u^{\prime}$ in $Z$ and nonzero polynomials $f_{1}(T), f_{2}(T), g_{1}(T), g_{2}(T) \in R^{\prime}[T]$ such that $c_{\delta}=f_{1}\left(u_{\delta}\right) / g_{1}\left(u_{\delta}\right)$ and $c_{\delta}=f_{2}\left(u_{\delta}^{\prime}\right) / g_{2}\left(u_{\delta}^{\prime}\right)$. But then the polynomial $f_{1}\left(T_{1}\right) g_{2}\left(T_{2}\right)-f_{2}\left(T_{2}\right) g_{1}\left(T_{1}\right) \in$ $R^{\prime}\left[T_{1}, T_{2}\right]$ is nonzero (because $c_{\delta} \notin R^{\prime}$ ) and shows that $\left\{u_{\delta}, u_{\delta}^{\prime}\right\}$-and hence $Z_{[\delta]}$-is not algebraically independent over $R^{\prime}$. To prove (14.1), we show

there is a subset $Z^{\prime}$ of $\Phi_{\beta} \cap V_{\beta}$ of cardinality $2^{\aleph_{0}}$ such that $Z_{[\delta]}^{\prime}$ is algebraically independent over $Y_{\delta}$.

This suffices, by the additivity of transcendence degree, since $\left|\left\{y_{\gamma}: \gamma<\alpha\right\}\right|<$ $2^{\aleph_{0}}$.

In order to prove (14.2), we write the complement of $\Phi_{\beta}$ as the union, $\bigcup_{n \in \omega} C_{n}$, of countably many closed nowhere dense sets in $\mathscr{T}_{Q / R}^{\delta}\left(N_{\beta}\right)$. Choose a ladder $\left\{\tau_{n}: n \in \omega\right\}$ on $\delta$ and inductively define a tree of basic clopen subsets $U_{\eta} \subseteq V_{\beta}\left(\eta \in{ }^{n} 2\right)$ so that $U_{\eta} \subseteq U_{\zeta}$ if $\zeta=\eta \uparrow l(\zeta)$ and $U_{\eta} \subseteq$ the complement of $C_{l(\eta)}$. Moreover, we choose the $U_{\eta}$ so that for each $n$, if ${ }^{n} 2=\left\{\eta_{1}, \ldots, \eta_{2^{n}}\right\}$, then there are ordinals $\gamma_{i}$ with $\tau_{n}<\gamma_{0}<\cdots<\gamma_{2^{n}}$ such that for every $z_{i} \in U_{\eta_{i}}\left(i=1, \ldots, 2^{n}\right)$ and $g \in G^{+}$, if $\gamma_{1} \leq g \leq \gamma_{2^{n}}$, then $g \in \operatorname{supp}\left(z_{i}\right)$ if and only if $g=\gamma_{i-1}$. Then one may prove by induction on $m$ that if $f\left(T_{1}, \ldots, T_{m}\right) \in Y_{\delta}\left[T_{1}, \ldots, T_{m}\right]$ is a nonzero polynomial whose coefficients are in $\widehat{R}_{\tau_{n}}$ and if $z_{j} \in U_{\eta_{j}}$, then $f\left(z_{1}, \ldots, z_{m}\right) \in \widehat{R}_{\gamma_{m+1}} \backslash\{0\}$. Hence if we choose for each $\xi \in{ }^{\omega} 2, z_{\xi} \in \bigcap_{n \in \omega} U_{\xi \mid n}$, then $Z_{[\delta]}=\left\{z_{\xi}: \xi \in{ }^{\omega} 2\right\}$ is algebraically independent over $Y_{\delta}$.

Finally consider the case when $\alpha=2 \beta$. It suffices to show that there is a subset $Z$ of $\mathscr{T}_{Q / R}^{\delta}\left(Y_{\delta}\right)$ of cardinality $2^{\aleph_{0}}$ so that $Z_{[\delta]}$ is algebraically independent over $Y_{\delta}$ and each $f \in Z$ has the form $\left\langle w_{\sigma}\left(u_{\sigma}^{\beta}\right)^{-1}: \sigma<\delta\right\rangle$ where $\left\langle w_{\sigma}: \sigma<\delta\right\rangle \in \mathscr{T}_{Q / R}^{\delta}\left(Y_{\delta}\right)$ and for each $\sigma<\delta, w_{\sigma} \in W_{\delta}$. Now by a tree argument similar to that above, there is a subset $Z^{\prime}$ of $\mathscr{T}_{Q / R}^{\delta}\left(Y_{\delta}\right)$ of cardinality $2^{\aleph_{0}}$ consisting of elements $w=\left\langle w_{\sigma}: \sigma<\delta\right\rangle$ such that for all $\sigma$ the coefficients of $w_{\sigma}$ belong to $\{0,1\}$ and such that $Z_{[\delta]}^{\prime}$ is algebraically independent over $Y_{\delta}$. Then by an argument on transcendence degree, there is a subset $S$ of $\left\{w_{\delta}\left(u^{\beta}\right)_{\delta}^{-1}: w \in Z^{\prime}\right\}$ of cardinality $2^{\aleph_{0}}$ which is algebraically independent over $Y_{\delta}$. So let

$$
Z=\left\{\left\langle w_{\sigma}\left(u_{\sigma}^{\beta}\right)^{-1}: \sigma<\delta\right\rangle: w_{\delta}\left(u^{\beta}\right)_{\delta}^{-1} \in S\right\}
$$

The following corollary shows that the converse of Theorem 7 is not provable in $\mathrm{ZFC}+\mathrm{GCH}$.

Corollary 15. ( $\mathrm{V}=\mathrm{L})$ There is a valuation domain $R$ of cardinality $\aleph_{1}$ such that $\Gamma_{R}^{\prime}(Q / R)=0$ and there is a nonstandard uniserial $R$-module of type $Q / R$. 
Proof. Let $R$ be the valuation domain constructed as in the proof of Theorem 14 for some stationary $S$. (The construction requires only $\mathrm{CH}$.) Then $\Gamma_{R}^{\prime}(Q / R)=0$. Also $\Gamma_{R}(Q / R)=\widetilde{S} \neq 0$, so by Proposition 3, there is a nonstandard uniserial $R$-module, since $\mathrm{V}=\mathrm{L}$, and hence $\diamond_{\omega_{1}}(S)$ holds.

\section{VALUATION DOMAINS OF CARDINALITY $>\aleph_{1}$}

Finally we want to observe that the hypothesis that $R$ has cardinality $\aleph_{1}$ is essential for Proposition 3; that is, it is not enough to require that $J$ is generated by $\aleph_{1}$ elements (in which case the definition of $\Gamma_{R}(J / A)$ makes sense). In fact, we have the following theorem of ZFC.

Theorem 16. There is a valuation domain $R$ of cardinality $2^{\aleph_{1}}$ such that $Q$ is generated (as $R$-module) by $\aleph_{1}$ elements and $\Gamma_{R}(Q / R)=1$, but every uniserial $R$-module of type $Q / R$ is standard.

Proof. Let $G$ be the free abelian group on $\omega_{1} \times 2^{\aleph_{1}}$. We order $\omega_{1} \times 2^{\aleph_{1}}$ lexicographically and then order $G$ antilexicograhically (cf. Definition 10). Thus in $G,(\alpha, i)<(\beta, j)$ if and only if $\alpha<\beta$ (in $\left.\omega_{1}\right)$ or $\alpha=\beta$ and $i<j$ (in $2^{\aleph_{1}}$ ) and in that case $n(\alpha, i)<(\beta, j)$ for all $n \in \mathbb{Z}$. Let $\widehat{R}=K[[G]]$ be defined as in Definition 10, for this $G$ and for a field $K$ of cardinality $\leq \aleph_{1}$. Define $p$-supp and truncation analogously to Definition 10.

Let $Y_{1}$ be the smallest valuation subring of $\widehat{R}$ containing $X^{(\alpha, 0)}$ for all $\alpha \in$ $\omega_{1}$ and let $r_{\nu}=X^{(\nu, 0)}$ for $\nu<\omega_{1}$. Then $Q=\bigcup_{\nu<\omega_{1}} r_{\nu}^{-1} R$ and $\Gamma_{Y_{1}}\left(Q / Y_{1}\right)=$ 1 .

Define $G_{i}$ to be the subgroup of $G$ generated by $\left\{(\alpha, j): \alpha<\omega_{1}, j<i\right\}$ and let $\widehat{R}_{i}$ be the subring of $\widehat{R}$ consisting of all $y \in \widehat{R}$ such that $\operatorname{supp}(y) \subseteq G_{i}$. We shall now define a continuous chain $\left\{Y_{i}: i<2^{\aleph_{1}}\right\}$ of valuation subrings of $R$ such that for each $i, Y_{i} \subseteq \widehat{R}_{i}$, and is closed under truncation-more precisely, if $y \in Y_{i}$ and $\delta \in \lim \left(\omega_{1}\right)$, then $y \nmid(\delta, 0) \in Y_{i}$. Moreover, we require that

$$
\text { for all } i<j, \quad Y_{j} \cap \widehat{R}_{i}=Y_{i} \text {. }
$$

We will let $R$ be the union of the $Y_{i}$. Then certainly $\Gamma_{R}(Q / R)=1$, since we will have added no limits of elements of $Y_{1}$. Moreover, we will do the construction so that for each family $\left\{e_{\sigma}^{\tau}: \sigma<\tau<\omega_{1}\right\}$ as in ( $\left.\dagger\right)$, there is a family $\left\{c_{\sigma}: \sigma<\omega_{1}\right\}$ as in $(\dagger \dagger)$. There are only $\left(2^{\aleph_{1}}\right)^{\aleph_{1}}=2^{\aleph_{1}}$ possible families $\left\{e_{\sigma}^{\tau}: \sigma<\tau<\omega_{1}\right\}$ and the cofinality of $2^{\aleph_{1}}>\aleph_{1}$, so we can arrange our construction such that each such family is considered at some stage $i<2^{\aleph_{1}}$ where $\left\{e_{\sigma}^{\tau}: \sigma<\tau<\omega_{1}\right\} \subseteq Y_{i}$.

Suppose now that $Y_{i}$ has been constructed and that at this stage we are considering the family $\left\{\boldsymbol{e}_{\sigma}^{\tau}: \sigma<\tau<\omega_{1}\right\} \subseteq Y_{i}$. Our plan is to let $Y_{i+1}$ be the smallest valuation subring of $\widehat{R}$ which is closed under truncation and contains $Y_{i} \cup\left\{X^{(\alpha, i)}: \alpha<\omega_{1}\right\}$ and a family $\left\{c_{\sigma}: \sigma<\omega_{1}\right\}$ as in (††); we must choose $\left\{c_{\sigma}: \sigma<\omega_{1}\right\}$ so that $(\mathfrak{E})$ holds. We define the $c_{\sigma}$ by induction. Let $c_{0}=1$; if $c_{\sigma}$ has been defined, let

$$
c_{\sigma+1}=e_{\sigma}^{\sigma+1} c_{\sigma}+X^{(\sigma, i)} .
$$

If $\delta$ is a limit ordinal, let $c_{\delta}$ be the unique element of $\widehat{R}$ with support in $\bigoplus_{j<i} \bigoplus_{\alpha<\omega_{1}} \mathbb{Z}(\alpha, j)$ which represents the limit of $\left\langle e_{\sigma}^{\delta} c_{\sigma}: \sigma<\delta\right\rangle$ in $\widehat{R} / \bigcap_{\sigma<\delta} r_{\sigma} \widehat{R}$. 
We sketch the argument that this construction works. First, by induction one can show that for all $\tau,(\tau, i) \in p-\operatorname{supp}\left(c_{\sigma}\right)$ if and only if $\tau<\sigma$. Secondly, the $c_{\sigma}$ are algebraically independent over the quotient field of $\hat{R}_{i}$. More generally, if $\sigma_{1}<\cdots<\sigma_{n}<\delta \leq \sigma_{n+1}$, then $\left\{c_{\sigma_{1}}, \ldots, c_{\sigma_{n}}, c_{\sigma_{n+1}} \uparrow \delta\right\}$ is algebraically independent over $\widehat{R}_{i}$. (This is proved by an argument on supports.) Now an arbitrary element of $Y_{i+1}$ has the form

$$
\frac{f\left(c_{\sigma_{1}}, \ldots, c_{\sigma_{n}}\right) \uparrow(\delta, 0)}{g\left(c_{\sigma_{1}}, \ldots, c_{\sigma_{n}}\right) \uparrow(\gamma, 0)}
$$

where $f$ and $g$ have coefficients in $Y_{i}$. By an argument on supports, using the above facts, one shows that if this element belongs to $Y_{i+1} \cap \widehat{R}_{i}$, then it belongs to $Y_{i}$.

\section{REFERENCES}

[BFS] S. Bazzoni, L. Fuchs, and L. Salce, The hierarchy of uniserial modules over a valuation domain, preprint.

[BS1] S. Bazzoni and L. Salce, On non-standard uniserial modules over valuation domains and their quotients, J. Algebra 128 (1990), 292-305.

[BS2] _ Elongations of uniserial modules over valuation domains, J. Algebra 146 (1992), 304317.

[BS3] _ Equimorphism classes of uniserial modules over valuation domains, Arch. Math. 58 (1992), 14-23.

[D] K. Devlin, Constructibility, Springer-Verlag, Berlin and New York, 1984.

[DSh] K. Devlin and S. Shelah, $A$ weak version of $\diamond$ which follows from $2^{\aleph_{0}}<2^{\aleph_{1}}$, Israel J. Math. 29 (1978), 239-247.

[E] P. Eklof, A transfer theorem for non-standard uniserials, Proc. Amer. Math. Soc. 114 (1992), 593-600.

[EM] P. Eklof and A. Mekler, Almost free modules, North-Holland, Amsterdam, 1990.

[FrG] B. Franzen and R. Göbel, Nonstandard uniserial modules over valuation domains, Resultate Math. 12 (1987), 86-94.

[FS] L. Fuchs and L. Salce, Modules over valuation domains, Marcel Dekker, New York, 1985.

[FSh] L. Fuchs and S. Shelah, Kaplansky's problem on valuation rings, Proc. Amer. Math. Soc. 105 (1989), 25-30.

[O1] B. L. Osofsky, Constructing nonstandard uniserial modules over valuation domains, Azumaya Algebras, Actions, and Modules, Contemp. Math., vol. 124, Amer. Math. Soc., Providence, RI, 1992, pp. 151-164

[O2] - A construction of nonstandard uniserial modules over valuation domains, Bull. Amer. Math. Soc. 25 (1991), 89-97.

[Sh] S. Shelah, Nonstandard uniserial module over a uniserial domain exists, Lecture Notes in Math., vol. 1182, Springer-Verlag, Berlin and New York, 1986, pp. 135-150.

Department of Mathematics, University of California, Irvine, California 92717

E-mail address: pceklof@uci.edu

Department of Mathematics, Hebrew University, Jerusalem, IsRael

E-mail address: shelah@math.huji.ac.il

Department of Mathematics, Rutgers University, New Brunswick, New Jersey 08903 